\title{
RESEARCH
}

Open Access

\section{Macrophage M2 polarization induced by exosomes from adipose-derived stem cells contributes to the exosomal proangiogenic effect on mouse ischemic hindlimb}

Dihan Zhu' ${ }^{1}$, Takerra K. Johnson², Yang Wang ${ }^{1}$, Miracle Thomas ${ }^{1}, K^{2}$ Huynh ${ }^{1}$, Qinglin Yang ${ }^{3}$, Vincent C. Bond ${ }^{4}$, Y. Eugene Chen ${ }^{5}$ and Dong Liu ${ }^{1,6^{*}}$

\begin{abstract}
Background: M2 macrophages and exosomes from adipose-derived stem cells (ASCs) are both reported to promote angiogenesis. However, the possible synergistic effects between exogenous exosomes and endogenous M2 macrophages are poorly understood.

Methods: Exosomes were isolated from conditioned medium of normoxic and hypoxic ASCs using the combined techniques of ultrafiltration and size-exclusion chromatography and were identified with nanoparticle tracking analysis and immunoblotting for exosomal markers. Macrophages were collected from the mouse peritoneal cavity. M1 and M2 macrophages were detected by immunoblotting for the intracellular markers inducible nitric oxide synthase (iNOS) and arginase-1 (Arg-1) and by flow cytometry for the surface markers F4/80, CD86, and CD206. Murine models of Matrigel plug and hindlimb ischemia were employed as in vivo angiogenic assays.

\footnotetext{
* Correspondence: dliu@msm.edu

${ }^{1}$ Cardiovascular Research Institute, Morehouse School of Medicine, 720

Westview Drive SW, Atlanta, GA 30310, USA

${ }^{6}$ Department of Physiology, Morehouse School of Medicine, Atlanta, GA, USA

Full list of author information is available at the end of the article
}

(C) The Author(s). 2020 Open Access This article is licensed under a Creative Commons Attribution 4.0 International License, which permits use, sharing, adaptation, distribution and reproduction in any medium or format, as long as you give appropriate credit to the original author(s) and the source, provide a link to the Creative Commons licence, and indicate if changes were made. The images or other third party material in this article are included in the article's Creative Commons licence, unless indicated otherwise in a credit line to the material. If material is not included in the article's Creative Commons licence and your intended use is not permitted by statutory regulation or exceeds the permitted use, you will need to obtain permission directly from the copyright holder. To view a copy of this licence, visit http://creativecommons.org/licenses/by/4.0/ The Creative Commons Public Domain Dedication waiver (http://creativecommons.org/publicdomain/zero/1.0/) applies to the data made available in this article, unless otherwise stated in a credit line to the data. 
(Continued from previous page)

Results: When M1 macrophages were treated with exosomes from normoxic ASCs (Nor/Exo), and particularly from hypoxic ASCs (Hyp/Exo), the expression of the M1 marker iNOS decreased, and the M2 marker Arg-1 increased in a time- and dosedependent manner. Additionally, a decrease in the M1 surface marker CD86 and an increase in the M2 surface marker CD206 were observed, which suggested that M1 macrophages were polarized to an M2-like phenotype. Conditioned medium from these M2-like macrophages presented lower levels of proinflammatory cytokines and higher levels of proangiogenic factors and promoted endothelial cell proliferation, migration, and tube formation. Furthermore, M2 polarization and angiogenesis were induced upon the administration of exosomes in mouse Matrigel plug and hindlimb ischemia (HLI) models. Interestingly, these exosomal effects were attenuated by using a colony stimulating factor 1 receptor (CSF-1R) inhibitor, BLZ945, in vitro and in vivo. Downregulation of microRNA-21 (miR-21) in hypoxic ASCs reduced the exosomal effects on M2 polarization, Akt phosphorylation, and CSF-1 secretion. A similar reduction in exosomal activity was also observed when exosomes were administered along with BLZ945.

Conclusion: Our findings provide evidence that exosomes from ASCs polarize macrophages toward an M2like phenotype, which further enhances the exosomal proangiogenic effects. Exosomal delivery of miR-21 and positive feedback of secreted CSF-1 may be involved in macrophage polarization.

Keywords: Exosome, Stem cells, Macrophage, Angiogenesis, microRNA

\section{Introduction}

Ischemic cardiovascular and cerebrovascular diseases continue to represent the most common cause of death in modern society. To address this concern, research on therapeutic angiogenesis has been evolving for the past decades [1]. The protein/gene approach, stem/progenitor cell approach, and subsequent microvesicle/exosome approach have been employed as therapies for ischemic diseases $[2,3]$. Adipose-derived stem cells (ASCs) possess the characteristics of easy acquisition, high proliferative potential, and low immunogenicity, making them attractive candidates for cell-based therapeutics [4,5]. Unlike living cells, exosomes are submicron vesicles that target recipient cells to deliver their cargo for cell-cell communication. Our laboratory and others have demonstrated that exosomes from ASCs have angiogenic effects $[5,6]$. However, the interrelationship between the administered exosome preparations and the innate reparative aspects in angiogenesis remain largely unexplored.

Ischemic diseases, similar to infectious diseases, activate macrophages and trigger inflammatory responses to injured/infarcted tissues [7, 8]. While M1 macrophages, a classically activated subset, function in the early inflammatory stages to stimulate the inflammatory response and clean cell debris, M2 macrophages, an alternatively activated subset, accumulate in the late inflammatory stages to alleviate the inflammatory response and initiate angiogenesis and tissue repair [9-12]. In view of excess M1 macrophages in the late stages leading to exacerbated lesions and delayed recovery, it is crucial for macrophages to be polarized to the M2 phenotype on time [11]. Traditionally, M2 polarization is driven by anti-inflammatory cytokines secreted by $\mathrm{T}$ helper 2 cells in the late stages of infection [13].
Stem cells, including ASCs, are recently reported to induce M2 polarization through secretomes when used for tissue repair or inflammatory inhibition $[14,15]$. Transfusion of mesenchymal stem cell (MSC)-educated M2 macrophages is reported to reduce tissue injury in a mouse acute kidney injury model. Severe histological and functional injury is observed if M1 macrophages are transfused [16]. Stem cellderived exosomes have been broadly studied as a cell-free therapeutic approach. Recently, exosomes from ASCs have been implicated in reducing inflammation in white adipose tissue for anti-obesity effects by polarizing M2 macrophages [17]. In the current study, we demonstrate that in addition to promoting angiogenesis directly, exosomes from hypoxic ASCs induce M1 to M2 macrophage transition, which further enhances the exosomal proangiogenic effects in ischemic hindlimbs. Colony stimulating factor 1 (CSF-1) secretion upregulated by exosomal microRNA-21 (miR-21) delivery may mediate and have positive feedback on the transition of the macrophage phenotype.

\section{Materials and methods \\ Cell culture}

Mouse adipose-derived stem cells (ASCs) were purchased from Cyagen (Santa Clara, CA). Mouse cardiac microvascular endothelial cells (CMVECs) were purchased from Cellbiologics (Chicago, IL). Both cell types were maintained at $37^{\circ} \mathrm{C}$ in a humidified $5 \% \mathrm{CO}_{2}$ incubator. Cells at passages 4-6 were used for all experiments.

\section{Exosome isolation}

ASCs were incubated in either normoxic or hypoxic (1\% $\mathrm{O}_{2}$ ) conditions in basal medium containing $1 \%$ exosomefree FBS for 3 days. Exosomes were isolated from the 
incubated medium using combination techniques of ultrafiltration and size-exclusion chromatography as described previously [18]. The protein concentration of the isolated exosomes was detected by using a total exosome RNA and Protein Isolation Kit (Thermo Fisher Scientific; Waltham, MA) and a BCA Protein Assay Kit (Thermo Fisher Scientific) according to the manufacturer's instructions. In all cases, the FBS used in this study was exosome-free FBS, which was obtained by ultracentrifugation of FBS at $100,000 \times g$ for $18 \mathrm{~h}$ at $4{ }^{\circ} \mathrm{C}$.

\section{Nanoparticle tracking analysis (NTA)}

An LM10 NTA device (Malvern; Amesbury, UK) was used according to the manufacturer's recommendations. Each exosome sample was analyzed by detecting the rate of the Brownian motion of particles in liquid suspension. The analysis settings were optimized, and each video was analyzed to obtain the mean, mode, median, and estimated concentration of each particle size. A total of $500 \mu \mathrm{l}$ of a 1:5-diluted exosome sample in phosphatebuffered saline (PBS) was injected into a NanoSight sample cubicle, which yielded a particle concentration of $1 \times$ $10^{8}$ particles $/ \mathrm{ml}$.

\section{Immunoblotting}

Immunoblotting was performed as previously described [6]. Proteins were detected using primary anti-CD9 (ab92726, Abcam, Cambridge, MA), anti-TSG101 (T5701, MilliporeSigma, St. Louis, MO), anti-Alix (ab186429, Abcam), anti-iNOS (13120S, Cell Signaling), anti-Arg-1 (93668S, Cell Signaling), anti-GAPDH $(437,000$, Thermo Fisher Scientific), anti-P (phospho)-Akt Ser473 (4060, Cell Signaling), anti-P-Akt Thr308 (13,038, Cell Signaling), and anti-Akt (4685, Cell Signaling) antibodies. Exposure of the resultant protein bands was performed with an ImageQuant LAS 4000 Luminescent Image Analyzer (GE Healthcare; Chicago, IL).

\section{Macrophage isolation, polarization, and polarizing inhibition}

All animal experiments in this study were approved by the Institutional Animal Care and Use Committee of Atlanta University Center and complied with the $\mathrm{NIH}$ guidelines for the care and use of laboratory animals. Male C57BL/6J mice at 6-8 weeks of age were purchased from The Jackson Laboratory (Bar Harbor, ME). Five milliliters of PBS was intraperitoneally injected after euthanasia. Diluted peritoneal fluid was withdrawn and loaded onto a 70- $\mu \mathrm{m}$ cell strainer (Corning; Corning, $\mathrm{NY}$ ). The filtrate was centrifuged at $250 \times g$ for $15 \mathrm{~min}$, and the cell pellet was resuspended in Macrophage-SFM (Thermo Fisher Scientific). The cell suspension was transferred to a cell culture vessel. The attached cells were defined as M0 macrophages. M1 polarization was induced by incubating M0 macrophages in MacrophageSFM supplemented with $10 \mu \mathrm{mol} / \mathrm{L} \quad$ IFN- $\gamma \quad$ (R\&D Systems, Minneapolis, MN) for $24 \mathrm{~h}$ and then with $10 \mu \mathrm{mol} / \mathrm{L}$ IFN- $\gamma$ plus $10 \mathrm{ng} / \mathrm{ml}$ LPS (MilliporeSigma) for another $24 \mathrm{~h}$. M2 polarization was induced by incubating M0 macrophages in Macrophage-SFM supplemented with $15 \mu \mathrm{mol} / \mathrm{L}$ IL-4 (R\&D Systems) and $10 \mu \mathrm{mol} / \mathrm{L} \mathrm{IL-}$ 10 (Thermo Fisher Scientific) for $48 \mathrm{~h}$ [19]. M2 macrophages were used as a positive control in this study. BLZ945 (Selleck Chemicals, Houston, TX), an M2 polarization inhibitor that inhibits colony stimulating factor 1 receptor (CSF-1R) [20, 21], was used at a concentration of $30 \mathrm{ng} / \mathrm{ml}$ along with exosomes when indicated.

\section{Exosome uptake}

Exosomes from ASCs were labeled using an ExoGlow-Protein EV Labeling Kit (System Biosciences; Palo Alto, CA) according to the manufacturer's instructions. M1 macrophages were incubated in the labeled exosome suspension at a concentration of $30 \mu \mathrm{g} / \mathrm{ml}$ in Macrophage-SFM for the indicated times. The cells were then counterstained with Hoechst 33342 (Thermo Fisher Scientific) and examined by fluorescence microscopy (Olympus, Japan) and flow cytometry [18].

\section{Flow cytometry analysis of macrophage markers}

M1 macrophages were incubated with $30 \mu \mathrm{g} / \mathrm{ml}$ exosomes for $48 \mathrm{~h}$. The cells were harvested and blocked with 3\% BSA in PBS for $30 \mathrm{~min}$, followed by incubation with FITC-anti-F4/80 and PE-anti-CD86 or PE-antiCD206 antibodies (BioLegend; San Diego, CA) in the dark. The cells were then analyzed by flow cytometry. The ExpressPlus program was selected. The cells incubated with FITC-isotype and PE-isotype (BioLegend) were used to exclude nonspecific binding by the fluorescent antibodies. M1 macrophages were defined as F4/ $80^{+}$and $\mathrm{CD}^{+} 6^{+}$. M2 macrophages were defined as $\mathrm{F} 4 /$ $80^{+}$and $\mathrm{CD}^{206^{+}}$.

\section{ELISA}

M1 macrophages were incubated with $30 \mu \mathrm{g} / \mathrm{ml}$ exosomes for $48 \mathrm{~h}$. The cells were then washed and incubated in fresh Macrophage-SFM for another $48 \mathrm{~h}$. The culture medium was collected and centrifuged at $500 \times g$ for $10 \mathrm{~min}$ at $4{ }^{\circ} \mathrm{C}$ to remove unadhered cells. The supernatant was used as conditioned medium (CdM). The concentrations of the cytokines and growth factors interleukin-4 (IL-4), IL-6, macrophage-CSF (M-CSF), granulocyte-macrophage-CSF (GM-CSF), vascular endothelial growth factor (VEGF), and basic fibroblast growth factor (bFGF) in the CdM were determined with 
corresponding ELISA kits (R\&D Systems) according to the manufacturer's instructions.

\section{Cell proliferation}

CMVECs were quiesced with endothelial basal medium/ $1 \% \mathrm{FBS}$ for $24 \mathrm{~h}$ and incubated in a $5 \mu \mathrm{mol} / \mathrm{L}$ fluorescent carboxyfluorescein succinimidyl ester (CFSE) solution (green) from the CellTrace CFSE Cell Proliferation Kit (Thermo Fisher Scientific) at $37^{\circ} \mathrm{C}$ for $20 \mathrm{~min}$. The cells were then washed and incubated in each of the various CdMs from macrophages as described above, buffered with an equal volume of endothelial basal medium/1\% FBS. The same medium was changed on day 2 . The cells were harvested at day 4 and subjected to analysis using a flow cytometer with 488-nm excitation and emission filters appropriate for fluorescein.

\section{Migration assay}

CMVECs were incubated in each of the various CdMs from macrophages as described above, buffered with an equal volume of endothelial basal medium/1\% FBS in a 24-well insert plate (BD Biosciences; San Jose, CA) at $37^{\circ} \mathrm{C}$ for $24 \mathrm{~h}$ according to the manufacturer's instructions. Cell nuclei were stained with Hoechst 33342. The cells that migrated to the lower side of the insert were counted under a fluorescence microscope as previously described [22].

\section{Tube formation assay}

CMVECs were seeded on plates precoated with growth factor-reduced Matrigel (BD Biosciences) and incubated in each of the various CdMs buffered with an equal volume of endothelial basal medium/1\% FBS as described above for $4 \mathrm{~h}$. The cells were then stained with Calcein AM (Thermo Fisher Scientific), and tube formation was visualized using fluorescence microscopy. Total vessel length, vessel area, number of junctions, and network complexity were calculated using AngioTool v.2 software [18].

\section{Matrigel plug assay}

Ice-cold Matrigel containing $100 \mu \mathrm{g}$ of exosomes from normoxic adipose-derived stem cells (Nor/Exo) or exosomes from hypoxic adipose-derived stem cells ( $\mathrm{Hyp} /$ Exo) at a volume of $250 \mu \mathrm{l}$ per plug was subcutaneously injected into the flanks of male, 6-8-week-old C57BL/6J mice (The Jackson Laboratory) as previously described [6]. The plugs were collected 2 weeks after implantation. One half of each plug was used for immunohistochemistry analysis, and the other half was digested with $2 \mathrm{mg} /$ $\mathrm{ml}$ collagenase type I in PBS containing $20 \mathrm{mM}$ HEPES at $37^{\circ} \mathrm{C}$ for $30 \mathrm{~min}$ and filtered to obtain the cell suspension for flow cytometry analysis as described above.

\section{Hindlimb ischemia model}

The femoral artery was ligated on the left hindlimb of male C57BL/6J mice (The Jackson Laboratory) at 6-8 weeks of age as reported [23]. The mice were subjected to intramuscular injection in the left adductor muscle [24] with $30 \mu \mathrm{g}$ of Nor/Exo or Hyp/Exo in $30 \mu \mathrm{l}$ of PBS immediately after ligation. The same intramuscular injections were performed at the surgery site 4 and 8 days post surgery. Blood perfusion on the plantar surface of the hind paws was measured immediately before and after surgery and 3, 7, 14, and 21 days post surgery using laser speckle imaging (LSI, Perimed AB, Järfälla, Sweden). The left to right ratio $(L / R)$ was used to represent the relative blood perfusion rate at the left hindlimb for each animal $(n=8)$. The mice were euthanized 3 weeks after the surgery. The left gastrocnemius muscle was collected for immunohistochemical analysis as described below. The left adductor muscle was also collected. One half was used for immunohistochemical analysis. The other half was cut into tiny pieces $\left(\sim 1 \mathrm{~mm}^{3}\right)$ and digested with $3 \mathrm{mg} / \mathrm{ml}$ collagenase type I in PBS containing $20 \mathrm{mM}$ HEPES at $37^{\circ} \mathrm{C}$ for $40 \mathrm{~min}$. The suspension was passed through a $70-\mu \mathrm{m}$ cell strainer (Corning). The filtered cells were used for flow cytometry analysis.

\section{Immunohistochemistry}

The cryosections of Matrigel plugs were stained with anti-CD31 antibody (AF3628, R\&D Systems) as previously described [6]. The muscle specimen samples were fixed, paraffin embedded, and sectioned. The sections were incubated with the primary anti- $\alpha$ smooth muscle actin ( $\alpha$-SMA, 19245, Cell Signaling) or anti-CD31 antibody (550,274, BD Biosciences) and counterstained with Hoechst 33342. The regions containing the most intense $\mathrm{CD}_{31}{ }^{+}$areas of neovascularization were chosen for quantification. Five hotspots per section and 3 sections per plug or muscle specimen were analyzed at $\times 400$ magnification. Image-Pro Plus software (Media Cybernetics) was used to measure $\mathrm{CD} 31^{+}$areas in each hotspot. Arteriole density was assessed as $\alpha$-SMA ${ }^{+}$vessels $/ \mathrm{mm}^{2}$.

\section{RNA isolation and microRNA (miRNA) profiling}

Total RNA was extracted from cells or exosomes using TRIzol Reagent (Thermo Fisher Scientific) according to the manufacturer's instructions. The miRNA profiling of extracted RNA was performed by LC Sciences (Houston, TX) $(n=2)$ using a microfluidic chip.

\section{RT-PCR for miRNAs}

Reverse transcription and quantitative real-time PCR were performed using the TaqMan miRNA assay system 
(Thermo Fisher Scientific) according to the manufacturer's instructions and our previous description [6]. The relative miRNA levels were normalized to endogenous U6 small nuclear RNA levels for each sample.

\section{Transduction of recombinant lentivirus}

The procedures performed here followed the National Institutes of Health guidelines for recombinant DNA research. All recombinant lentiviruses used in this study were purchased from Amsbio (Abingdon, UK). For transduction, ASCs were incubated with Lenti/ZipmiR21 (ZipmiR-21) or Lenti/ZipmiR-Control (ZipmiR-Cont) at an MOI of 2 in the presence of $8 \mu \mathrm{g} / \mathrm{ml}$ polybrene for $48 \mathrm{~h}$ before further treatment.

\section{Statistical analysis}

Statistical significance between the two groups was evaluated with a two-tailed Student's $t$ test. For multiple group comparisons (Matrigel plug assay and hindlimb ischemic model), one-way ANOVA followed by Tukey's multiple comparison test was performed. All values are reported as the mean \pm SD. Significance was accepted at the level of $p<0.05$.

\section{Results}

Exosomes from ASCs, particularly from hypoxic ASCs, polarize M1 macrophages to the M2-like phenotype

Exosomes are microparticles secreted from cells in sizes ranging from 30 to $150 \mathrm{~nm}$ [25]. In this study, NTA was used to determine the size distribution profile and relative particle density. The Hyp/Exo samples were within the expected range (Fig. 1a), and the Nor/Exo sample displayed a similar size distribution (data not shown). The amount of protein was used for exosome quantification. The higher protein amount (Fig. 1b) and higher levels of the exosomal markers CD9, TSG-101, and Alix (Fig. 1c) from the same number of hypoxic ASCs than normoxic ASCs suggested that ASCs secreted more exosomes upon hypoxia stimulation. To examine the polarization effect of the exosomes on M1 macrophages, M0 macrophages were collected from the peritoneal cavity of wild-type mice and subsequently polarized to M1 macrophages with standard cytokine treatment [19]. A typical pattern of the M1 marker iNOS and M2 marker Arg-1 determined by immunoblotting demonstrated the successful establishment of primary M1 macrophages along with the M2 macrophages used as a control in the following experiments (Figure I in the online-only Data

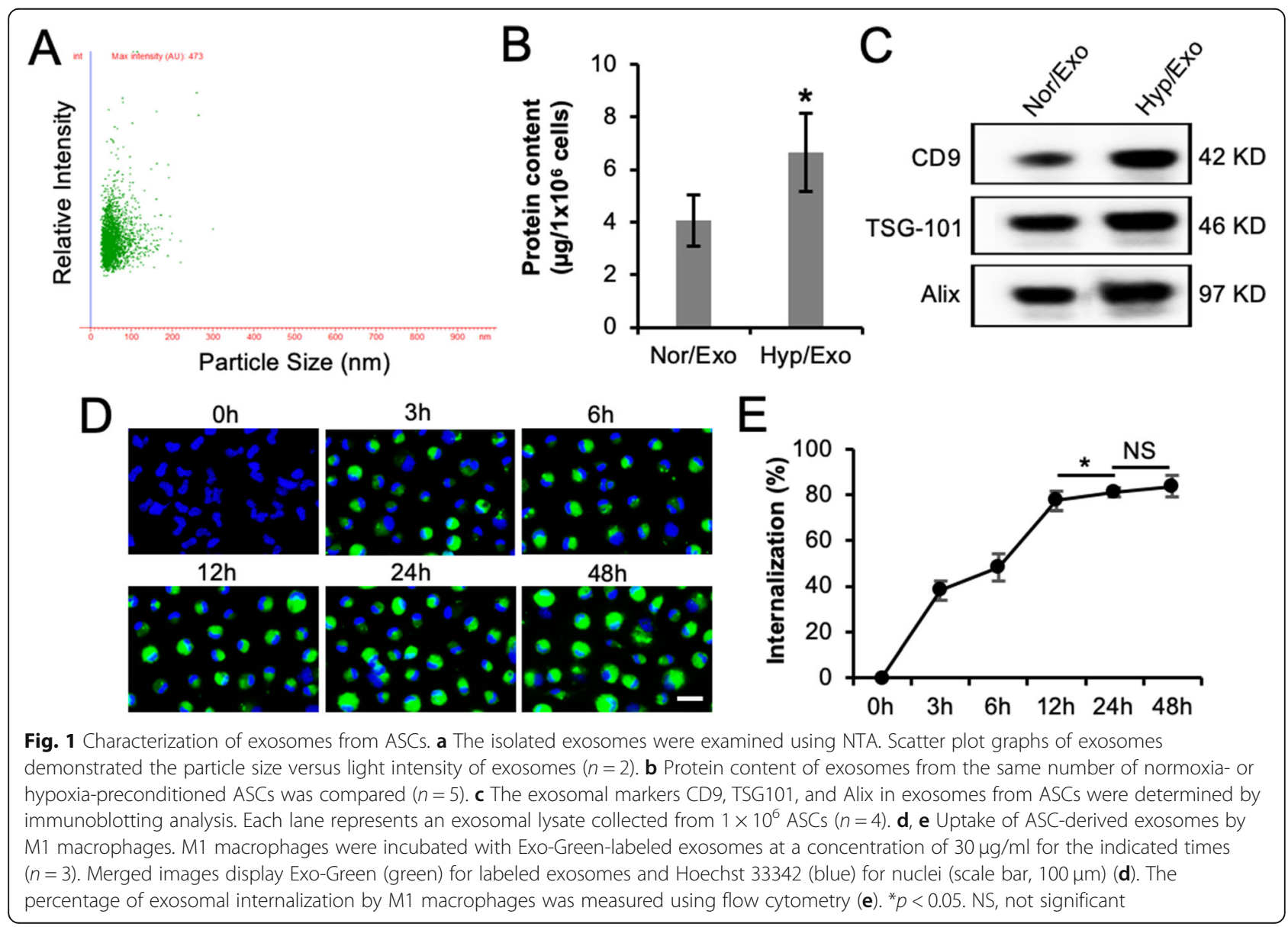




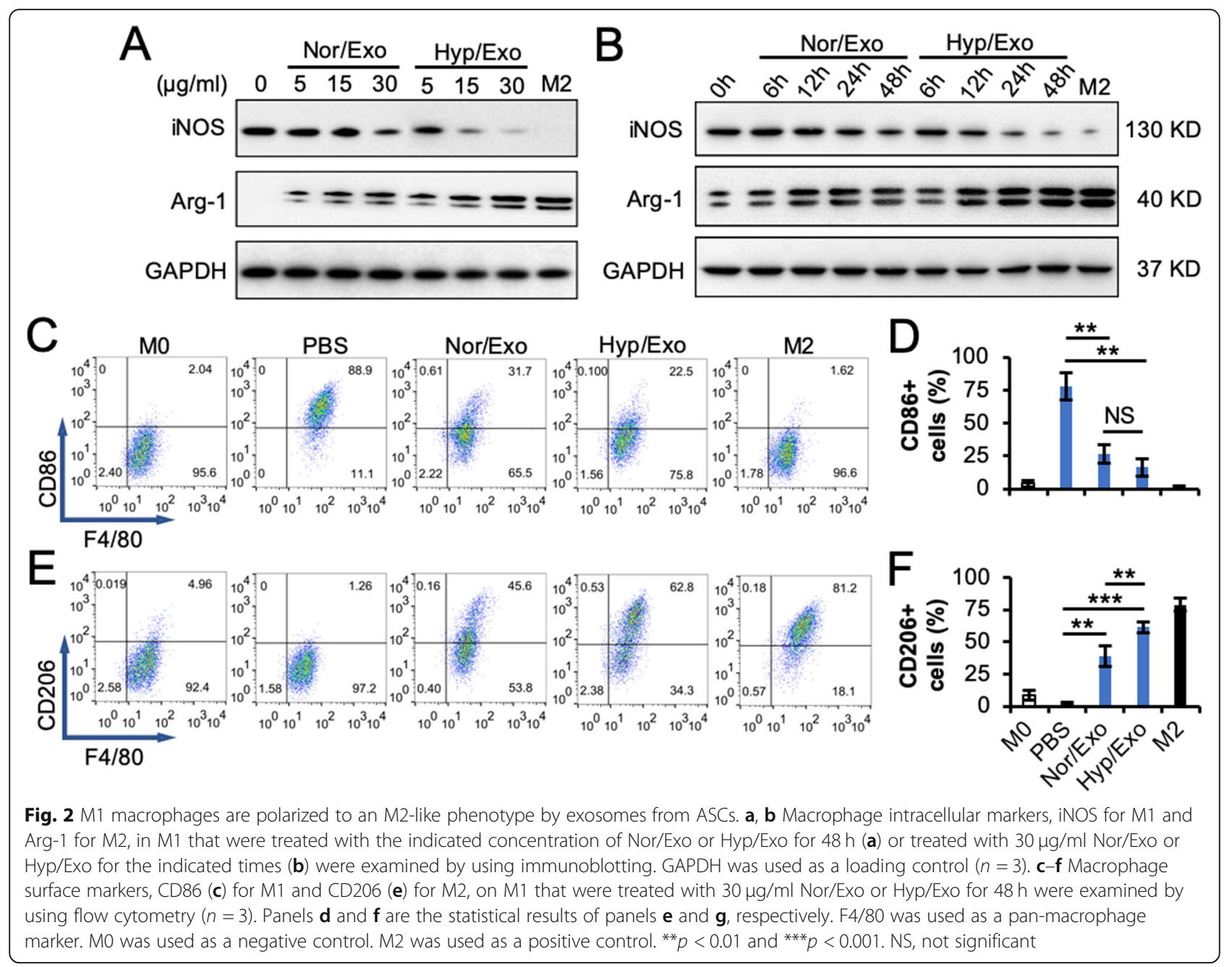

Supplement). A time course experiment of exosome uptake by M1 macrophages was performed to investigate the uptake efficiency. An increase in Hyp/Exo internalization into M1 macrophages with prolonged incubation time was observed in fluorescent images (Fig. 1d). The flow cytometry analysis revealed that the percentage of uptake gradually increased up to $85 \%$ in $48 \mathrm{~h}$ (Figure II in the online-only Data Supplement and Fig. 1e). The uptake of Nor/Exo by M1 macrophages was similar to that of Hyp/Exo (data not shown).

Upon the treatment of M1 macrophages with Nor/Exo or Hyp/Exo, iNOS was downregulated in a dose- (Fig. 2a) and time- (Fig. 2b) dependent manner, particularly when the cells were treated with Hyp/Exo. The opposite phenomenon was observed for Arg-1 when M1 macrophages were subjected to the same treatment. In addition, upon treatment of M1 macrophages with Nor/ Exo or Hyp/Exo, the M1 surface marker CD86 was downregulated, while the M2 surface marker CD206 was upregulated (Fig. 2c-f). Hyp/Exo showed a stronger impact on the changes in surface markers compared with Nor/Exo. These data suggest that exosomes from ASCs promote the polarization of M1 macrophages into M2like macrophages.

To investigate whether a similar polarization effect exists in human cells, human M1 macrophages were treated with Hyp/Exo from human ASCs. Flow cytometry analysis showed that CD80 (human M1 surface marker)-positive cells were decreased, while CD163 (human M2 surface marker)-positive cells were increased (Figure IIIA and IIIB in the online-only Data Supplement). Immunoblotting analysis showed that STAT1 (human M1 intracellular marker) was decreased and STAT3 (human M2 intracellular marker) was increased (Figure IIIC in the online-only Data Supplement). These observations suggest that human M1 macrophages could also be polarized to M2-like macrophages by exosomes from human ASCs. 


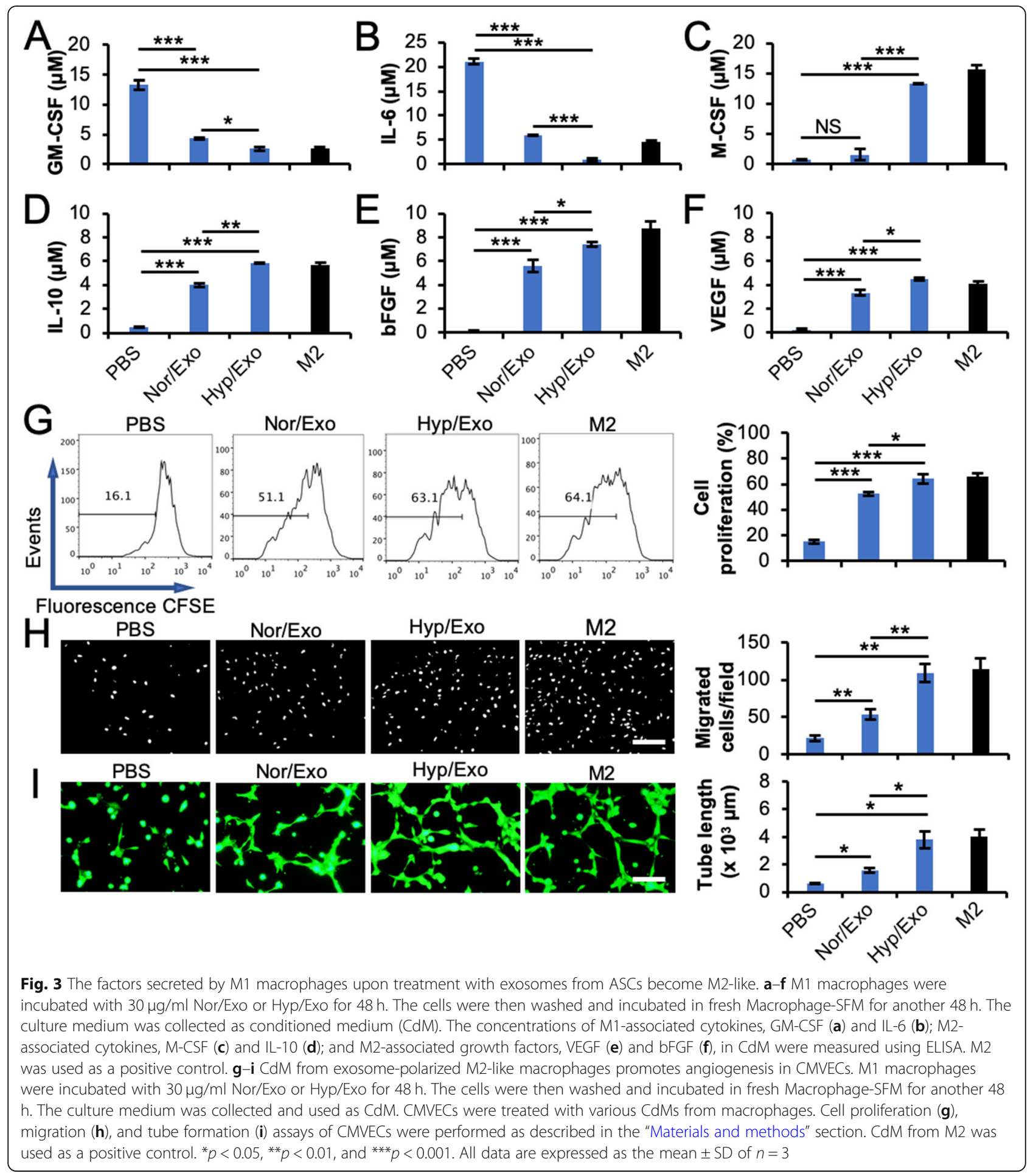

Proangiogenic effects of exosome-induced M2-like macrophages in vitro

It has been reported that M1 macrophages secrete proinflammatory factors, such as GM-CSF, IL-6, TNF, IL-1b, and IL-17, while M2 macrophages secrete antiinflammatory and proangiogenic factors, such as M-CSF,
IL-10, IL-4, TGF- $\beta$, bFGF, and VEGF $[11,19,26]$. To investigate the cytokines and growth factors secreted by our exosome-induced M2-like macrophages, CdM was collected from these cells and subjected to ELISA analysis. The results demonstrated that the concentrations of the cytokines GM-CSF (Fig. 3a) and IL-6 (Fig. 3b) were 


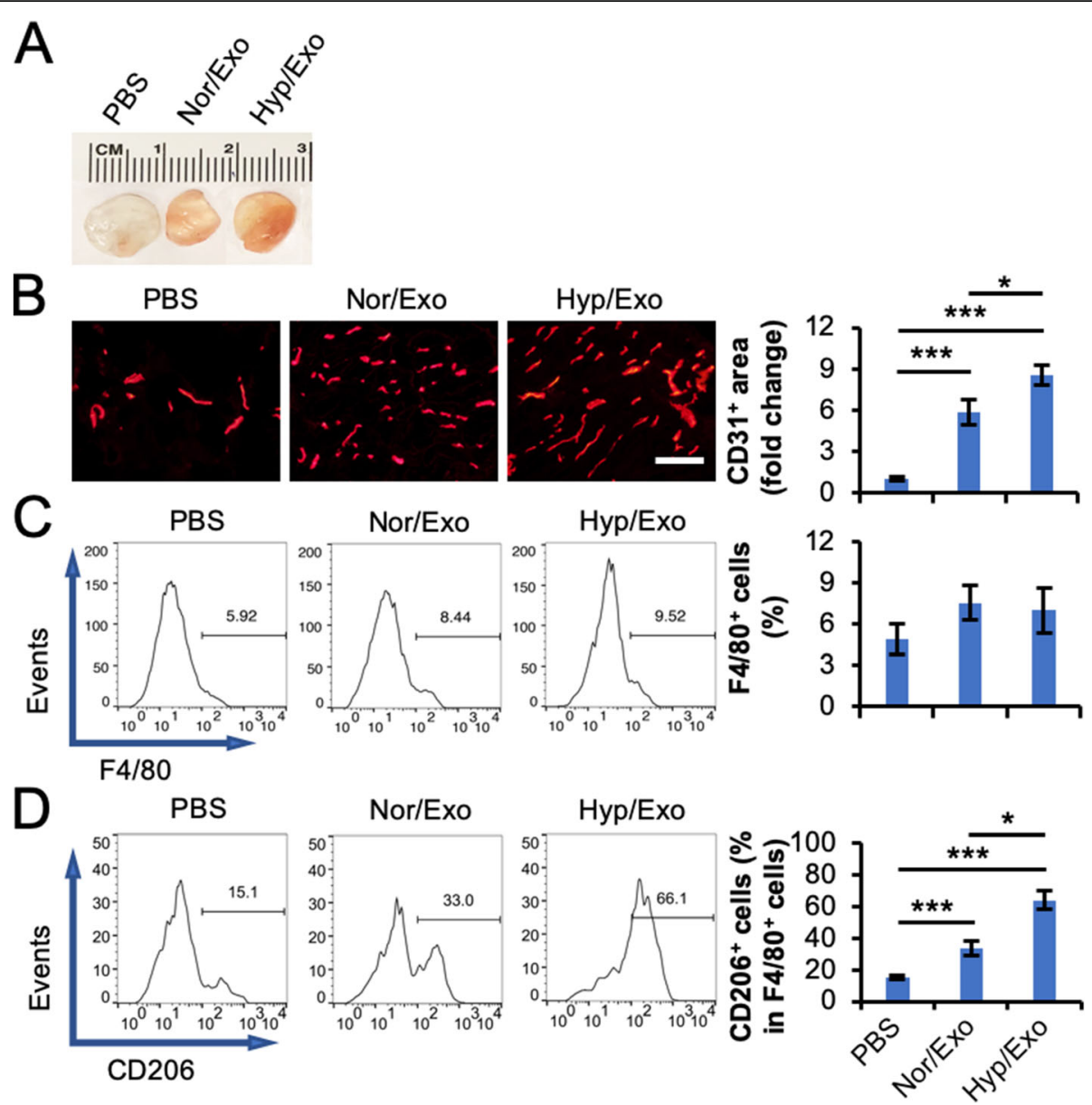

Fig. 4 Exosomes from ASCs promote angiogenesis in a mouse Matrigel plug assay. Matrigel containing Nor/Exo or Hyp/Exo was subcutaneously injected into the flanks of mice $(n=6)$. The Matrigel plugs were harvested 2 weeks post implantation. a Representative images of the plugs are shown. $\mathbf{b}$ The sections of the plugs were subjected to immunohistochemistry analysis for CD31, an endothelial cell marker. Scale bar $=100 \mu \mathrm{m}$. Quantification of the $\mathrm{CD}_{3} 1^{+}$area was performed. The positive area in the slide with plugs containing PBS was set to 1. c, d Cells collected from the plugs were subjected to flow cytometry analysis. The percentage of $\mathrm{F} / 80^{+}$cells in the total analyzed cells is displayed (c). The percentage of $\mathrm{CD}^{206} 6^{+}$, an M2 macrophage marker, cells in F4/80 ${ }^{+}$cells was further analyzed (d). ${ }^{*} p<0.05$ and ${ }^{* *} p<0.01$

decreased, while M-CSF (Fig. 3c) and IL-10 (Fig. 3d) as well as the growth factors bFGF (Fig. 3e) and VEGF (Fig. 3f) were increased in CdM from M1 macrophages upon treatment with Nor/Exo or Hyp/Exo. These data confirm the impact of exosomes from ASCs on M2 polarization and imply an angiogenic function of polarized M2-like macrophages. To investigate the angiogenic effect, CdM was therefore used to treat CMVECs. The results showed that CdM from Nor/Exo- or Hyp/Exo-treated M1 macrophages promoted CMVEC proliferation (Fig. 3g), migration (Fig. 3h) and tube formation (Fig. 3i and Figure IVA through IVC in the online-only Data Supplement). Our findings provide evidence that Nor/Exo, particularly Hyp/Exo, induced M2-like macrophages to secrete more angiogenic cytokines and growth factors as well as to promote CMVEC angiogenesis in vitro.

\section{Exosome-induced angiogenesis and M2 polarization in} mouse Matrigel plug and HLI models

To study the role of exosomes from ASCs in angiogenesis and M2 polarization under pathophysiological conditions, a mouse Matrigel plug assay was conducted. Our results revealed that Nor/Exo or Hyp/Exo enhanced functional vasculature formation, as indicated by the red appearance of the plugs (Fig. 4a) and the CD31-positive area in the plug section (Fig. 4b). The number of pan macrophages which defined by F4/80-positive was not affected by either Nor/Exo or Hyp/Exo (Fig. 4c). However, the percentage of CD206-positive cells in F4/80positive cells was increased in the plugs containing Nor/ Exo or Hyp/Exo (Fig. 4d). These results provide evidence that ASC-derived exosomes, especially hypoxiapreconditioned ASC-derived exosomes, promote 


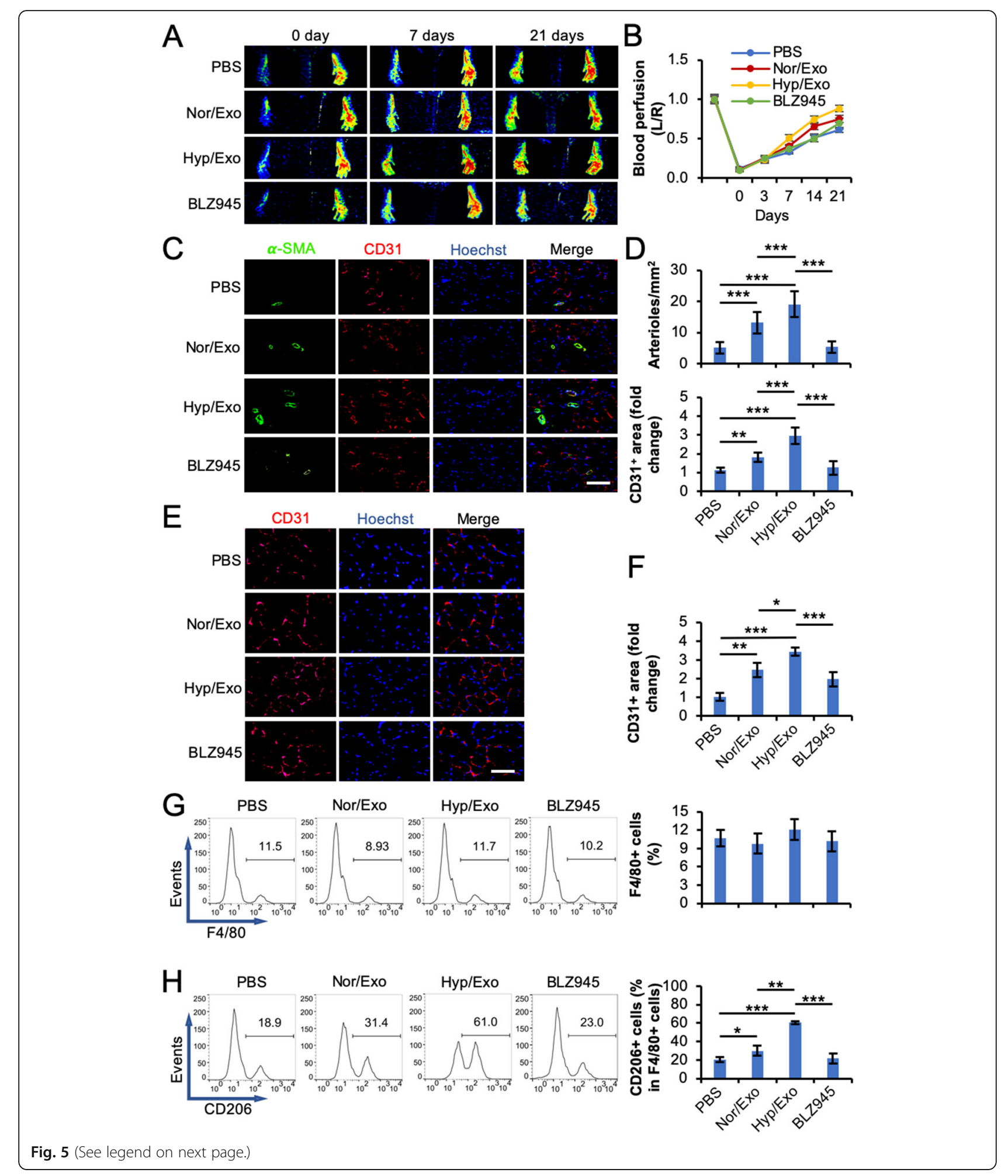


(See figure on previous page.)

Fig. 5 Exosomes from ASCs promote angiogenesis and M2 polarization in a mouse HLI model. After left femoral artery ligation, PBS, Nor/ExO, Hyp/Exo, or Hyp/Exo plus BLZ945 (BLZ945), an M2 polarization inhibitor that targets CSF-1R, was intramuscularly injected $(n=8)$. a Representative laser speckle images showing the different recovery of blood perfusion in the hind paws on the indicated days. b Quantitative analyses of the images showing the left/right ratio of plantar perfusion. c-f, The mice were euthanized 3 weeks post surgery. The sections of the adductor muscle from the ligated side were subjected to immunohistochemistry analysis for a-SMA, a smooth muscle marker, and CD31, an endothelial cell marker, and counterstained with Hoechst 33342 (c, scale bar $150 \mu \mathrm{m}$ ). Quantification of the arteriole number per square millimeter and the $\mathrm{CD}_{1} 1^{+}$area (d). The sections of the gastrocnemius muscle from the ligated side were subjected to immunohistochemistry analysis for CD31 and counterstained with Hoechst $33342\left(\mathbf{e}\right.$, scale bar $100 \mu \mathrm{m}$ ). Quantification of the CD31 $1^{+}$area $(\mathbf{f})$. The $\mathrm{CD} 31^{+}$area on the slide from the mouse administered PBS was set to 1. g Flow cytometric analysis of the percentage of F4/80 cells in cells dissociated from the adductor muscle. $\mathbf{h}$ The percentage of $\mathrm{CD}_{20}{ }^{+}$cells in the F4/80 ${ }^{+}$cell population. GAPDH was used as a loading control. ${ }^{*} p<0.05,{ }^{* *} p<0.01$, and ${ }^{* * *} p<0.001$

angiogenesis and increase the proportion of M2 macrophages in the plug.

A mouse hindlimb ischemia (HLI) model was also used to explore the effects of exosomes on angiogenesis and macrophage polarization [23]. The results demonstrated that blood perfusion in the hind paw was enhanced by the administration of Nor/Exo starting from day $14(p<0.05)$ or Hyp/Exo starting from day $7(p<$ $0.05)$ post femoral artery ligation in comparison with perfusion with the administration of PBS (Fig. 5a and b). The results of immunohistochemistry analysis showed that the vascular smooth muscle cell marker $\alpha$-SMA was elevated by Nor/Exo, particularly by Hyp/Exo in the adductor muscle (Fig. 5c and d). Similar increases were observed for the vascular endothelial cell marker CD31 in the adductor muscle (Fig. $5 \mathrm{c}$ and $\mathrm{d}$ ) and the gastrocnemius muscle (Fig. 5e and f). Interestingly, the administration of BLZ945, which is reported to block M2 polarization by inhibiting the activation of CSF-1R [20, 21], was observed to decrease Hyp/Exo-upregulated expression of $\alpha$-SMA and CD31. To explore the phenotype of macrophages at the ligation site, the macrophages in the adductor muscle were analyzed by flow cytometry. The data showed that upon treatment with either Nor/ Exo or Hyp/Exo, the percentage of F4/80-positive macrophages in the total analyzed cells from the adductor muscle was unchanged (Fig. 5g). However, the percentage of CD206-positive cells in the F4/80-positive macrophages was increased upon the administration of Nor/ Exo or Hyp/Exo (Fig. 5h). Similar to the in vitro observation above, BLZ945 impaired the polarizing effect of Hyp/Exo on macrophages in the adductor muscle. These data suggest that Nor/Exo or Hyp/Exo from ASCs promote arteriogenesis and angiogenesis, which may be partially through CSF-1R-induced M2 polarization (but not macrophage recruitment) in this animal model.

\section{Exosomes from hypoxic ASCs induce M2 polarization via transport of miR-21}

As our results above demonstrated that Hyp/Exo had a greater impact on M2 polarization than Nor/Exo, miRNA profiling was performed for Nor/Exo and Hyp/ Exo to define the underlying molecular mechanisms of
M2 polarization induced by exosomes from ASCs (Figure V in the online-only Data Supplement). The profiling analysis identified 501 exosomal miRNAs with a ratio of Hyp/Exo to Nor/Exo greater than 2:1 (Fig. 6a). Based on an extensive review of reported M2 polarization-regulating miRNAs [12, 27-31], seven of the 501 miRNAs were considered to promote M2 polarization. The seven miRNAs, miR-21, miR-223, Let7c, miR-146b, miR-33, miR-511-3p, and miR-181a-5p, were validated by RT-PCR analysis (Fig. 6b). Among these miRNAs, miR-21 showed the greatest increase in Hyp/Exo compared with Nor/Exo. Downregulation of miR-21 in Hyp/Exo was achieved by transducing Lenti/ ZipmiR-21 into donor hypoxic ASCs (Fig. 6c). When M1 macrophages were treated with miR-21-knockdown Hyp/Exo (ZipmiR-21), the extent of the decrease in iNOS expression and the increase in Arg-1 expression and Akt (Ser473 and Th308) phosphorylation by the control Hyp/Exo (ZipmiR-Cont) were reduced (Fig. 6d). The effects of Hyp/Exo on downregulating the percentage of CD86-positive macrophages and upregulating the percentage of CD206-positive macrophages were also reduced by knockdown of miR-21 in Hyp/Exo (Fig. 6e). The concentrations of M-CSF (Fig. 6f) and IL-10 (Fig. 6g) in the CdM of M1 macrophages treated with miR-21knockdown Hyp/Exo were much lower than those in the group treated with the control Hyp/Exo. When BLZ945 was used along with Hyp/Exo, similar effects to those of miR-21-knockdown Hyp/Exo were observed (Fig. 6d-g). These results suggest that miR-21 in Hyp/Exo from ASCs plays an important role in exosome-induced M2 polarization by regulating Akt phosphorylation.

\section{Discussion}

As a critical component of innate immunity, macrophages regulate the balance between inflammatory activity and tissue repair [32]. The polarization of macrophages to the M2 phenotype promotes angiogenesis and is a potential therapeutic approach to ischemic diseases [10-12]. Our previous studies have shown that exosomes from ASCs directly target vascular endothelial cells to promote angiogenesis [6]. Here, we demonstrate 

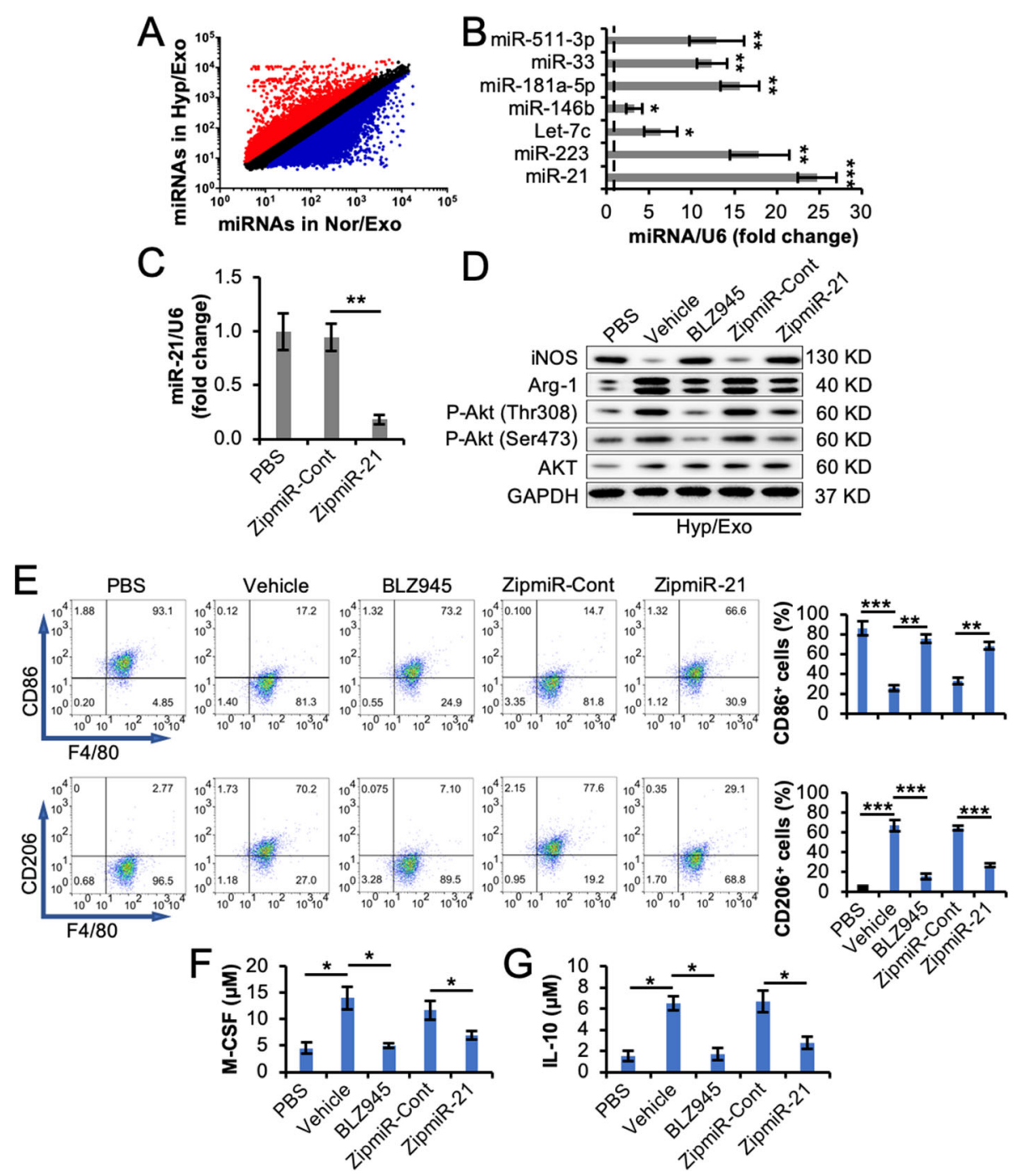

Fig. 6 miR-21 enriched in Hyp/Exo from ASCs contributed to M2 polarization. a Profiling of miRNAs in Nor/Exo and Hyp/Exo was performed $(n=$ 2). The relative average levels of each miRNA in Nor/Exo and Hyp/Exo are shown. Each dot represents one miRNA. The miRNAs with a ratio of Hyp/Exo to Nor/Exo greater than 2 are marked red, and those less than 0.5 are marked blue. All the other miRNAs in between are marked black. b The levels of miRNAs that satisfy both marked red in panel (a) and reported to promote M2 polarization were further examined with RT-PCR analysis. c Downregulation of miR-21 in Hyp/Exo from ASCs. Hypoxic ASCs were untransduced (PBS) or transduced with Lenti/ZipmiR-Cont (ZipmiR-Cont) or with Lenti/ZipmiR-21 (ZipmiR-21) to knockdown miR-21. Hyp/Exo secreted from these cells were isolated. The level of miR-21 in the different Hyp/Exo groups was examined with RT-PCR to verify the downregulation. U6 was used as an internal control. $\mathbf{d}-\mathbf{g}$ M1 macrophages were treated with PBS or Hyp/Exo in the presence of BLZ945 or miR-21-silenced Hyp/Exo (ZipmiR-21). Vehicle and ZipmiR-Cont were used as controls for BLZ945 and ZipmiR-21, respectively. The levels of iNOS, Arg-1, P-Akt (Ser473), and P-Akt (Thr308) in the treated M1 macrophages were examined by immunoblotting analysis. GAPDH and Akt (pan) were used as loading controls (d). The percentage of CD86 ${ }^{+}$or CD206 ${ }^{+}$cells among $\mathrm{F} 4 / 80^{+}$cells in the treated M1 macrophages was quantified by using flow cytometry analysis (e). The concentrations of M-CSF (f) and IL-10 (g) in the CdM of the treated M1 macrophages were examined by using ELISA. ${ }^{*} p<0.05$, ${ }^{* *} p<0.01$, and ${ }^{* * *} p<0.001$. All data are expressed as the mean \pm SD of $n=3$

that exosomes enhance proangiogenic effects by targeting macrophages to polarize the M1 to M2 phenotype.

ASC-derived exosomes were recently implied to reduce inflammation in white adipose tissue through reprogramming adipose macrophages from the M1 to M2 subtype [17]. We provide evidence that exosomes from ASCs, particularly from hypoxic ASCs, induce mouse
M1 macrophage skewing to an M2-like phenotype partially by activating CSF-1R. Exosomes from hypoxiatreated human ASCs enhance endothelial cell tube formation [33]. Hypoxia incubation is a mimic of the ischemic circumstance of cells. ASCs secrete more exosomes under ischemia, which implies a release of more signals requiring more blood or oxygen supply. We 
further revealed that exosomes from hypoxic human ASCs induce human monocyte-derived M1 macrophages to be polarized to an M2-like phenotype. Compared with M1 macrophages, our data show that M2like macrophages polarized by exosomes from ASCs secrete more angiogenic factors and that $\mathrm{CdM}$ from M2like macrophages promotes endothelial cell proliferation, migration, and tube formation. We and other laboratories have reported that exosomes from ASCs promote angiogenesis [4-6, 33]. However, the possible synergistic effects between exogenous proangiogenic exosomes and endogenous proangiogenic M2 macrophages are poorly understood. Our findings demonstrate that exosomes from hypoxic ASCs do not increase the total number of macrophages but do increase the percentage of the M2 population while enhancing arteriogenesis, angiogenesis, and blood perfusion in mouse angiogenic models. The administration of the CSF-1R inhibitor BLZ945 affects the efficacy of exosomes in the ischemic hindlimb. A recent study demonstrated that exosomes from bone marrow-derived MSCs attenuate myocardial ischemiareperfusion injury through macrophage polarization, which is consistent with our observations [34].

miRNAs in exosomes play an essential role in mediating cell-cell communication. Based on an extensive review of the literature, two dozen miRNAs were found to induce macrophage M2 polarization [34, 35]. Among them, seven miRNAs were enriched in Hyp/Exo from ASCs, namely, miR-21, miR-223, Let-7c, miR-146b, miR-181a, miR-33, and miR-511-3p, according to our miRNA array results. miR-21 was validated as having the greatest increase and was shown to contribute to the induction of macrophage M2 polarization by Hyp/Exo from ASCs. According to the Ingenuity Pathway Analysis prediction, $80 \%$ of miR-21 targets are associated with macrophage polarization toward an inflammatory phenotype, whereas only $10 \%$ are associated with the M2 phenotype. Inhibition of miR-21 in macrophages has been demonstrated to reduce macrophage M2 polarization [12, 27-31]. Exosomes from miR-21-downregulated cancer cells have also been observed to indirectly mitigate macrophage M2 polarization [36]. However, in the mouse macrophage cell line RAW264.7, the miR-21 mimic attenuates M2 polarization, while the miR-21 inhibitor augments M2 polarization [35]. This opposite observation may be caused by the immortalization and transformation of the cell line.

miR-21 targets phosphatase and tensin homolog (PTEN), which inhibits the phosphoinositide 3-kinase (PI3K)/Akt pathway [37]. As one of the most extensively studied signaling pathways, the PI3K/Akt pathway is involved in almost every aspect of macrophage activation [38, 39]. An Akt allosteric inhibitor suppresses Akt phosphorylation and M2 polarization [40]. Similarly, our results show that Hyp/Exo with downregulated miR-21 or together with the CSF-1 inhibitor BLZ945 decreases Akt phosphorylation, M2 polarization, and cytokine secretion, including M-CSF. miR-21 is reported to elevate CSF-1 secretion through PTEN downregulation and Akt phosphorylation in breast tumors [41]. CSF-1 binds to CSF-1R, reinforces PI3K/Akt activation, and promotes M2 polarization, which can be blocked by a CSF-1R inhibitor [20, 42]. These observations imply that CSF-1 autocrine/paracrine signaling is critical in exosomal miR-21-induced PI3K/Akt pathway activation and M2 polarization as a positive feedback (Figure VI in the online-only Data Supplement). However, miR-21 is unlikely to be the only factor responsible for exosomes from hypoxic ASCs inducing M2 polarization. Other miRNAs enriched in Hyp/Exo, either reported to be macrophage polarization-related or not, may also have the capacity to promote M2 polarization. In addition, exosomes from ASCs carry a variety of proteins that are biologically active and can trigger functional responses in target cells [20]. Indeed, exosomal proteins from tumors have been recently reported to modulate M2 macrophage polarization [43]. Additional studies regarding the effects of proteins in exosomes from hypoxic ASCs on M2 polarization may be needed to further elucidate the underlying molecular mechanisms.

\section{Conclusion}

In summary, our results reveal that exosomes derived from ASCs induce M1 macrophages from mice or humans to be polarized to a proangiogenic M2-like phenotype. Hypoxic preconditioning in ASCs enhances the quantity of secreted exosomes as well as the ability of secreted exosomes to promote angiogenesis and M2 polarization, which can be reduced by a CSF-1R inhibitor. Exosomal delivery of miR-21 contributes to M2-like polarization and the secretion of proangiogenic cytokines, including CSF-1. The PI3K/Akt pathway may mediate miR-21 delivery-induced CSF-1R activation and M2 polarization. This study uncovers a novel underlying mechanism of exosomes derived from hypoxic ASCs as a therapeutic strategy for ischemic diseases and expands our understanding of the role of the immune system in the response to therapeutic angiogenesis. The effect of exosomes on regulating the polarization of macrophages may also imply their broader application for certain immune diseases by modulating immune responses and promoting tissue repair.

\section{Supplementary information}

Supplementary information accompanies this paper at https://doi.org/10. 1186/s13287-020-01669-9.

\section{Additional file 1.}




\section{Abbreviations}

Arg-1: Arginase-1; ASC: Adipose-derived stem cell; bFGF: Basic fibroblast growth factor; CFSE: Carboxyfluorescein succinimidyl ester; CMVEC: Cardiac microvascular endothelial cell; CSF-1: Colony stimulating factor 1; CSF1R: Colony stimulating factor 1 receptor; GM-CSF: Granulocyte-macrophagecolony stimulating factor; HLI: Hindlimb ischemia; Hyp/Exo: Exosomes from hypoxic adipose-derived stem cells; IL: Interleukin; iNOS: Inducible nitric oxide synthase; MSC: Mesenchymal stem cell; miRNA/miR: MicroRNA; MCSF: Macrophage-colony stimulating factor; Nor/Exo: Exosomes from normoxic adipose-derived stem cells; PTEN: Phosphatase and tensin homolog; VEGF: Vascular endothelial growth factor; ZipmiR-21: Lenti/ZipmiR21; ZipmiR-Cont: Lenti/ZipmiR-Control

\section{Acknowledgements}

We thank Dr. Ming-Bo Huang for the assistance with nanoparticle tracking analysis, Dr. Yan Xiao for the assistance with immunohistochemistry and Dr. Babayewa Oguljahan for the technical assistance with animal surgery.

\section{Authors' contributions}

Dihan Zhu: Collection and/or assembly of data, data analysis and interpretation, manuscript writing. Takerra K. Johnson: Collection and/or assembly of data. Yang Wang: Collection and/or assembly of data. Miracle Thomas: Collection and/or assembly of data. Ky Huynh: Collection and/or assembly of data. Qinglin Yang: Conception and design. Vincent C. Bond: Conception and design. Y. Eugene Chen: Conception and design. Dong Liu: Conception and design, financial support, data analysis and interpretation, manuscript writing, final approval of the manuscript. The authors read and approved the final manuscript.

\section{Funding}

This work was supported by National Heart, Lung, and Blood Institute (NHLBI) grants SC1HL134212 (to D.L.) and P5OHL117929 (Director Herman A. Taylor, Sub-Project Principal Investigator D.L.) and a National Institute on Minority Health and Health Disparities grant G12MD007602 (Director Vincent C. Bond, Sub-Project Principal Investigator D.L.).

\section{Availability of data and materials}

The data that support the findings of this study are available from the corresponding author upon reasonable request.

\section{Ethics approval and consent to participate}

All animal experiments in this study were approved by the Institutional Animal Care and Use Committee of the Atlanta University Center and complied with the $\mathrm{NIH}$ guidelines for the care and use of laboratory animals.

\section{Consent for publication}

Not applicable.

\section{Competing interests}

The authors indicate no potential conflicts of interest.

\section{Author details}

${ }^{1}$ Cardiovascular Research Institute, Morehouse School of Medicine, 720 Westview Drive SW, Atlanta, GA 30310, USA. ${ }^{2}$ Ophthalmic Genetics and Visual Function Branch, National Eye Institute, Bethesda, MD, USA. ${ }^{3}$ Department of Pharmacology, Louisiana State University School of Medicine, New Orleans, LA, USA. ${ }^{4}$ Department of Microbiology, Biochemistry \& Immunology, Morehouse School of Medicine, Atlanta, GA, USA. ${ }^{5}$ Department of Internal Medicine, University of Michigan Medical Center, Ann Arbor, MI, USA. ${ }^{6}$ Department of Physiology, Morehouse School of Medicine, Atlanta, GA, USA.

\section{Received: 26 December 2019 Revised: 10 March 2020}

\section{Accepted: 1 April 2020 Published online: 22 April 2020}

\section{References}

1. Potente M, Gerhardt H, Carmeliet P. Basic and therapeutic aspects of angiogenesis. Cell. 2011;146(6):873-87.

2. Tongers J, Losordo DW, Landmesser U. Stem and progenitor cell-based therapy in ischaemic heart disease: promise, uncertainties, and challenges. Eur Heart J. 2011;32(10):1197-206.

3. Johnson T, Zhao L, Manuel G, Taylor H, Liu D. Approaches to therapeutic angiogenesis for ischemic heart disease. J Mol Med (Berl). 2019;97(2):141-51.
4. Madonna R, Geng YJ, De Caterina R. Adipose tissue-derived stem cells: characterization and potential for cardiovascular repair. Arterioscler Thromb Vasc Biol. 2009;29(11):1723-9.

5. Zhao L, Johnson T, Liu D. Therapeutic angiogenesis of adipose-derived stem cells for ischemic diseases. Stem Cell Res Ther. 2017;8(1):125.

6. Kang T, Jones TM, Naddell C, Bacanamwo M, Calvert JW, Thompson WE Bond VC, Chen YE, Liu D. Adipose-derived stem cells induce angiogenesis via microvesicle transport of miRNA-31. Stem Cells Transl Med. 2016;5(4): 440-50.

7. Oliveira JB, Soares A, Sposito AC. Inflammatory response during myocardial infarction. Adv Clin Chem. 2018:84:39-79.

8. Planas AM. Role of immune cells migrating to the ischemic brain. Stroke. 2018;49(9):2261-7.

9. Mosser DM, Edwards JP. Exploring the full spectrum of macrophage activation. Nat Rev Immunol. 2008:8(12):958-69.

10. Takeda Y, Costa S, Delamarre E, Roncal C, Leite de Oliveira R, Squadrito ML, Finisguerra V, Deschoemaeker S, Bruyere F, Wenes M, et al. Macrophage skewing by Phd2 haplodeficiency prevents ischaemia by inducing arteriogenesis. Nature. 2011;479(7371):122-6.

11. Jetten N, Verbruggen S, Gijbels MJ, Post MJ, De Winther MP, Donners MM. Anti-inflammatory M2, but not pro-inflammatory M1 macrophages promote angiogenesis in vivo. Angiogenesis. 2014;17(1):109-18.

12. Ganta VC, Choi MH, Kutateladze A, Fox TE, Farber CR, Annex BH. A MicroRNA93-interferon regulatory factor-9-immunoresponsive gene-1itaconic acid pathway modulates M2-like macrophage polarization to revascularize ischemic muscle. Circulation. 2017;135(24):2403-25.

13. Sica A, Mantovani A. Macrophage plasticity and polarization: in vivo veritas. J Clin Invest. 2012;122(3):787-95.

14. Ben-Mordechai T, Holbova R, Landa-Rouben N, Harel-Adar T, Feinberg MS, Abd Elrahman I, Blum G, Epstein FH, Silman Z, Cohen S, et al. Macrophage subpopulations are essential for infarct repair with and without stem cell therapy. J Am Coll Cardiol. 2013;62(20):1890-901.

15. Shang Q, Bai Y, Wang G, Song Q, Guo C, Zhang L, Wang Q. Delivery of adipose-derived stem cells attenuates adipose tissue inflammation and insulin resistance in obese mice through remodeling macrophage phenotypes. Stem Cells Dev. 2015;24(17):2052-64.

16. Geng Y, Zhang L, Fu B, Zhang J, Hong Q, Hu J, Li D, Luo C, Cui S, Zhu F, et al. Mesenchymal stem cells ameliorate rhabdomyolysis-induced acute kidney injury via the activation of M2 macrophages. Stem Cell Res Ther. 2014;5(3):80.

17. Zhao H, Shang Q, Pan Z, Bai Y, Li Z, Zhang H, Zhang Q, Guo C, Zhang L, Wang Q. Exosomes from adipose-derived stem cells attenuate adipose inflammation and obesity through polarizing M2 macrophages and beiging in white adipose tissue. Diabetes. 2018:67(2):235-47.

18. Johnson TK, Zhao L, Zhu D, Wang Y, Xiao Y, Oguljahan B, Zhao X, Kirlin WG, Yin L, Chilian WM, et al. Exosomes derived from induced vascular progenitor cells promote angiogenesis in vitro and in an in vivo rat hindlimb ischemia model. Am J Physiol Heart Circ Physiol. 2019;317(4): H765-76.

19. Murray PJ, Allen JE, Biswas SK, Fisher EA, Gilroy DW, Goerdt S, Gordon S, Hamilton JA, Ivashkiv LB, Lawrence T, et al. Macrophage activation and polarization: nomenclature and experimental guidelines. Immunity. 2014; 41(1):14-20.

20. Pyonteck SM, Akkari L, Schuhmacher AJ, Bowman RL, Sevenich L, Quail DF, Olson OC, Quick ML, Huse JT, Teijeiro V, et al. CSF-1R inhibition alters macrophage polarization and blocks glioma progression. Nat Med. 2013; 19(10):1264-72

21. Yeh YM, Hsu SJ, Lin PC, Hsu KF, Wu PY, Su WC, Chang JY, Shen MR. The C. $1085 \mathrm{~A}>\mathrm{G}$ genetic variant of CSF1R gene regulates tumor immunity by altering the proliferation, polarization, and function of macrophages. Clin Cancer Res. 2017;23(20):6021-30.

22. Liu D, Hou J, Hu X, Wang X, Xiao Y, Mou Y, De Leon H. Neuronal chemorepellent Slit2 inhibits vascular smooth muscle cell migration by suppressing small GTPase Rac1 activation. Circ Res. 2006:98(4):480-9.

23. Limbourg A, Korff T, Napp LC, Schaper W, Drexler H, Limbourg FP. Evaluation of postnatal arteriogenesis and angiogenesis in a mouse model of hind-limb ischemia. Nat Protoc. 2009;4(12):1737-46.

24. Xie D, Li Y, Reed EA, Odronic SI, Kontos CD, Annex BH. An engineered vascular endothelial growth factor-activating transcription factor induces therapeutic angiogenesis in ApoE knockout mice with hindlimb ischemia. J Vasc Surg. 2006:44(1):166-75. 
25. Ribeiro MF, Zhu H, Millard RW, Fan GC. Exosomes function in pro- and antiangiogenesis. Curr Angiogenes. 2013;2(1):54-9.

26. Garg K, Pullen NA, Oskeritzian CA, Ryan JJ, Bowlin GL. Macrophage functional polarization (M1/M2) in response to varying fiber and pore dimensions of electrospun scaffolds. Biomaterials. 2013;34(18):4439-51.

27. Essandoh K, Li Y, Huo J, Fan GC. MiRNA-mediated macrophage polarization and its potential role in the regulation of inflammatory response. Shock. 2016;46(2):122-31.

28. Wu XQ, Dai Y, Yang Y, Huang $C$, Meng XM, Wu BM, Li J. Emerging role of microRNAs in regulating macrophage activation and polarization in immune response and inflammation. Immunology. 2016;148(3):237-48.

29. Roy S. miRNA in macrophage development and function. Antioxid Redox Signal. 2016;25(15):795-804.

30. Self-Fordham JB, Naqvi AR, Uttamani JR, Kulkarni V, Nares S. MicroRNA: dynamic regulators of macrophage polarization and plasticity. Front Immunol. 2017;8:1062.

31. Li H, Jiang T, Li MQ, Zheng XL, Zhao GJ. Transcriptional regulation of macrophages polarization by MicroRNAs. Front Immunol. 2018;9:1175.

32. Gordon S. Alternative activation of macrophages. Nat Rev Immunol. 2003; 3(1):23-35.

33. Xue C, Shen Y, Li X, Li B, Zhao S, Gu J, Chen Y, Ma B, Wei J, Han Q, et al. Exosomes derived from hypoxia-treated human adipose mesenchymal stem cells enhance angiogenesis through the PKA signaling pathway. Stem Cells Dev. 2018;27(7):456-65

34. Zhao J, Li X, Hu J, Chen F, Qiao S, Sun X, Gao L, Xie J, Xu B. Mesenchymal stromal cell-derived exosomes attenuate myocardial ischaemia-reperfusion injury through miR-182-regulated macrophage polarization. Cardiovasc Res. 2019;115(7):1205-16.

35. Ren W, Hou J, Yang C, Wang H, Wu S, Wu Y, Zhao X, Lu C. Extracellular vesicles secreted by hypoxia pre-challenged mesenchymal stem cells promote non-small cell lung cancer cell growth and mobility as well as macrophage M2 polarization via miR-21-5p delivery. J Exp Clin Cancer Res. 2019;38(1):62.

36. Caescu Cl, Guo X, Tesfa L, Bhagat TD, Verma A, Zheng D, Stanley ER. Colony stimulating factor-1 receptor signaling networks inhibit mouse macrophage inflammatory responses by induction of microRNA-21. Blood. 2015;125(8): e1-13.

37. Li J, Xie J, Liu S, Li X, Zhang D, Wang X, Jiang J, Hu W, Zhang Y, Jin B, et al. ADAR1 attenuates allogeneic graft rejection by suppressing miR-21 biogenesis in macrophages and promoting M2 polarization. FASEB J. 2018; 32(9):5162-73.

38. Meng F, Henson R, Wehbe-Janek H, Ghoshal K, Jacob ST, Patel T. MicroRNA21 regulates expression of the PTEN tumor suppressor gene in human hepatocellular cancer. Gastroenterology. 2007;133(2):647-58.

39. Cantley LC, Neel BG. New insights into tumor suppression: PTEN suppresses tumor formation by restraining the phosphoinositide 3-kinase/AKT pathway. Proc Natl Acad Sci U S A. 1999;96(8):4240-5.

40. Fruman DA, Chiu H, Hopkins BD, Bagrodia S, Cantley LC, Abraham RT. The PI3K pathway in human disease. Cell. 2017;170(4):605-35.

41. Byles V, Covarrubias AJ, Ben-Sahra I, Lamming DW, Sabatini DM, Manning BD, Horng T. The TSC-mTOR pathway regulates macrophage polarization. Nat Commun. 2013;4:2834.

42. Ries $\mathrm{CH}$, Cannarile MA, Hoves S, Benz J, Wartha K, Runza V, Rey-Giraud F, Pradel LP, Feuerhake F, Klaman I, et al. Targeting tumor-associated macrophages with anti-CSF-1R antibody reveals a strategy for cancer therapy. Cancer Cell. 2014;25(6):846-59.

43. Madonna R, Angelucci S, Di Giuseppe F, Doria V, Giricz Z, Gorbe A, Ferdinandy P, De Caterina R. Proteomic analysis of the secretome of adipose tissue-derived murine mesenchymal cells overexpressing telomerase and myocardin. J Mol Cell Cardiol. 2019;131:171-86.

\section{Publisher's Note}

Springer Nature remains neutral with regard to jurisdictional claims in published maps and institutional affiliations.

Ready to submit your research? Choose BMC and benefit from:

- fast, convenient online submission

- thorough peer review by experienced researchers in your field

- rapid publication on acceptance

- support for research data, including large and complex data types

- gold Open Access which fosters wider collaboration and increased citations

- maximum visibility for your research: over $100 \mathrm{M}$ website views per year

At BMC, research is always in progress.

Learn more biomedcentral.com/submissions 